\title{
SPARSE RECONSTRUCTION OF INCOMPLETE RELATIVE TRANSFER FUNCTION: DISCRETE AND CONTINUOUS TIME DOMAIN
}

\author{
Zbyněk Koldovský and Petr Tichavský \\ Faculty of Mechatronics, Informatics, and Interdisciplinary Studies, Technical University of Liberec, \\ Studentská 2, 46117 Liberec, Czech Republic. E-mail: zbynek.koldovsky@tul.cz
}

\begin{abstract}
Relative transfer functions (RTF) between microphones can often be estimated accurately but only for certain frequencies. For example, this happens in situations where the RTF is estimated from a noise-free signal of a target source whose spectrum does not span the whole frequency range. By combining a conventional RTF estimator and a selection of the active frequencies, an incomplete measurement of the RTF is obtained. We propose to retrieve the whole RTF estimate through finding the sparsest representation of the incomplete measurement in the discrete or continuous time-domain and compare both approaches. The RTF estimates are evaluated in terms of attenuation rate that measures the target signal cancellation at the output of a blocking matrix. It is shown by experiments that the reconstructed estimate can achieve significantly better attenuation than the initial (complete) estimate.
\end{abstract}

Index Terms - Relative Transfer Function, Blocking Matrix, Generalized Sidelobe Canceller, Sparse Approximation, LASSO, Atomic Norm, Semidefinite programming

\section{INTRODUCTION}

Two-microphone noisy recordings of a target source can be described as

$$
\begin{aligned}
& x_{\mathrm{L}}(n)=s(n)+z_{\mathrm{L}}(n), \\
& x_{\mathrm{R}}(n)=\left\{h_{\text {rel }} * s\right\}(n)+z_{\mathrm{R}}(n)
\end{aligned}
$$

where $n$ is the discrete-time index, $*$ denotes the convolution, $x_{\mathrm{L}}$ and $x_{\mathrm{R}}$ are, respectively, the signals from the left and right microphone, $s$ is the response of the target signal on the left microphone, and $z_{\mathrm{L}}$ and $z_{\mathrm{R}}$ are the other signals referred to as noise. The filter $h_{\text {rel }}$ denotes the relative impulse response (ReIR) between the microphones related to the target source. It is determined by the target's position, mutual position of the microphones, and it depends on the acoustical environment.

The estimation of $h_{\text {rel }}$ from noisy recordings is a key step to solve many challenging tasks of multichannel audio signal

This work was supported by The Czech Sciences Foundation through Project No. 14-11898S. processing $[1,2]$. For example, $h_{\text {rel }}$ can be used to construct an effective filter that cancels the target signal. Let its output be defined as

$$
\begin{aligned}
z(n) & =\left\{\hat{h} * x_{\mathrm{L}}\right\}(n)-x_{\mathrm{R}}(n) \\
& \approx\left\{h_{\mathrm{rel}} * z_{\mathrm{L}}\right\}(n)-z_{\mathrm{R}}(n) .
\end{aligned}
$$

The latter approximation follows from (1) when $\hat{h} \approx h_{\text {rel }}$. Hence, the output signal $z(n)$ provides key information (reference) about noise signals in (1), which is crucial, e.g., in noise reduction systems [3]. The residual signal-to-noise ratio measured in $z(n)$ reflects the degree of the target signal attenuation and is therefore a relevant criterion to validate $\hat{h}$.

The frequency-domain counterpart of $h_{\text {rel }}$ is referred to as Relative Transfer Function (RTF) and will be denoted as $H_{\mathrm{RTF}}(\theta), \theta \in[0,2 \pi)$. The problems of estimating $h_{\text {rel }}$ or $H_{\mathrm{RTF}}$ are equivalent tasks from this general point of view.

The practical requirement is to estimate the RTF from short intervals of recordings, because the target may change position and also the environment can be changing (air temperature, objects positions, noise). Therefore, the model (1), in which $h_{\text {rel }}$ is constant over time, is typically valid only during short intervals of length below one second. Several estimators have been proposed, some of which admit presence of noise signals in the recordings [1, 2, 4]. Especially, methods based on blind source separation can be used [5, 6]. However, the problem is still challenging as $h_{r e l}$ is typically long (has thousands taps) and dense. Some methods aim to learn a model of RTFs that were measured for different positions of the target source within a confined area [7, 8].

Recently, a new concept has been proposed in [9] based on using incomplete measurement of the RTF, which is drawing on ideas of Compressed Sensing [11]. The incomplete RTF (iRTF) is an RTF estimate whose values are known only for some frequencies. The iRTF can be obtained, for example, through applying any conventional RTF estimator where some of the estimated values are ignored. A reason to leave out an estimated value could be low target-to-noise ratio for the given frequency.

Then, the task to reconstruct the whole RTF poses an inverse problem. To solve, the fact that ReIRs are fast decaying (compressible) sequences is used. Such sequences, al- 
though being long and dense, can be efficiently approximated by sparse surrogates; see, e.g., [10]. Convex programming based on $\ell_{1}$-norm minimization is used to find the optimum sparse representation.

In this paper, we propose to reconstruct the incomplete RTF through finding its sparse representation in the continuous-time domain and compare it with the reconstruction done in the (oversampled) discrete time domain.

\section{PROBLEM FORMULATION}

We will consider a model where $h_{\text {rel }}$ is a linear combination of $S$ digital fractional delay filters, that is

$$
h_{\mathrm{rel}}(n)=\sum_{\ell=1}^{S} a_{\ell} \operatorname{sinc}\left(n-d_{\ell}\right), \quad n \in \mathcal{Z},
$$

where $\operatorname{sinc}(n)=\sin (\pi n) /(\pi n), a_{\ell} \in \mathcal{R}, d_{\ell} \in[0, L-1]$ for $\ell=1, \ldots, S$. Here, $S$ is not known in advance but is assumed to be much smaller than $L$. It means that $h_{\text {rel }}$ is sparse in the domain of fractional-delay filters.

The time-domain model (3) involves the assumption that the target signal (the main wave) and its reflections arrive at microphones with fractional time differences and that these reflections are sparse. Although natural sound propagation is typically more complicated, the model appears to be approximately valid under the hypothesis of ideal reflections (rigid walls/objects); see, e.g., [12, 13].

Without loss on generality, let the delays $d_{\ell}$ be all different and $L$ be even. In the special case that the delays are all integers, $h_{\text {rel }}$ is a finite sequence of maximum length $L$. Otherwise, $h_{\text {rel }}$ is infinite. The Discrete-Time Fourier Transform (DTFT) of (3) gives the assumed model of the RTF, that is,

$$
H_{\mathrm{RTF}}(\theta)=\sum_{\ell=1}^{S} a_{\ell} e^{-i d_{\ell} \theta} .
$$

Conventional methods operating in the Discrete Fourier Transform (DFT) domain estimate the RTF on the regular grid of $[0,2 \pi)$. Let $\widehat{H}_{\mathrm{RTF}}\left(\theta_{k}\right)$ denote such estimate of $H_{\mathrm{RTF}}\left(\theta_{k}\right)$ for the DFT length $L ; \theta_{k}=2 \pi k / L, k=0, \ldots, L-1$. $\widehat{H}_{\mathrm{RTF}}\left(\theta_{k}\right)$ is conjugate symmetric along $k=L / 2$, so the values for $k=L / 2+1, \ldots, L-1$ do not carry any extra information. We emphasize this fact, because the estimator in Section 3.1 does not necessarily obey this symmetry, respecting the fact that the values of $H_{\mathrm{RTF}}\left(\theta_{k}\right)$ obey the symmetry only if $h_{\text {rel }}$ is finite.

An estimate of the RFT is referred to as incomplete measurement if $\widehat{H}_{\mathrm{RTF}}\left(\theta_{k}\right)$ is available only for $k \in \mathcal{S} \subsetneq$ $\{0, \ldots, L / 2\}$. The central goal of this paper is to reconstruct the unknown values $H_{\mathrm{RTF}}\left(\theta_{k}\right)$ for $k \notin \mathcal{S}$ in some optimum sense given only the incomplete measurement. The goal could also be to improve (denoise) the estimates $\widehat{H}_{\mathrm{RTF}}\left(\theta_{k}\right)$ for $k \in \mathcal{S}$, but here we focus only on the former task due to limited space.

\section{PROPOSED SOLUTIONS}

Recent advances in convex programming bring new methods to find sparse representation of incomplete data in a given (possibly infinite) set of atoms called the atomic set, denoted as $\mathcal{A}$. The authors of [14] proposed a general framework based on atomic norm minimization. The atomic norm is a special case of the Minkowski functional measuring distance between a point and the convex hull of $\mathcal{A}$. It was shown that a linearly (or also quadratically [15]) constrained minimization of the atomic norm, with high probability, yields solutions that can be formed as a sum of a few atoms.

The linearly constrained optimization program is, in general, formulated as

$$
\min _{\mathbf{x}}\|\mathbf{x}\|_{\mathcal{A}} \quad \text { w.r.t. } \quad \mathbf{y}=\Phi \mathbf{x}
$$

where $\Phi$ stands for a measuring matrix, $\mathbf{y}$ is the (incomplete) measurement, and the atomic norm is defined as

$$
\|\mathbf{x}\|_{\mathcal{A}}=\inf \{t>0 \mid \mathbf{x} \in t \operatorname{conv}(\mathcal{A})\}
$$

where $\operatorname{conv}(\mathcal{A})$ denotes the convex hull of $\mathcal{A}$.

In this paper, we apply this general idea to the problem of reconstructing the RTF.

\subsection{Continuous Domain Reconstruction}

Let $\mathbf{x}$ represent the reconstructed $\mathrm{RTF}$, that is $x_{k+1}=$ $H_{\mathrm{RTF}}\left(\theta_{k}\right), k=0, \ldots, L-1$. The available iRTF is represented by $\mathbf{y}$, that is $y_{k+1}=\widehat{H}_{\mathrm{RTF}}\left(\theta_{j_{k}}\right)$ where $\mathcal{S}=$ $\left\{j_{1}, \ldots, j_{|\mathcal{S}|}\right\} . \Phi$ is equal to the $L \times L$ identity matrix $\mathbf{I}$ with only those rows whose indices are in $\mathcal{S}$; let denote such submatrix with the subscript $\cdot \mathcal{S}$, that is, $\Phi=\mathbf{I}_{\mathcal{S}}$.

The set $\mathcal{A}$ is defined as such that it contains transfer functions of all fractional delay filters and their complex-unit multiples (due to symmetry of $\mathcal{A}$ ), that is

$$
\mathcal{A}=\left\{e^{i \phi}\left[1 e^{i \theta} \ldots e^{i(L-1) \theta}\right]^{T}, \theta \in[0,2 \pi], \phi \in[0,2 \pi]\right\} .
$$

As expected by the theory, the solution of (5) should be a linear combination of a few elements of $\mathcal{A}$. This reflects the requirement that $S$ in (3) is "small".

The problem considered here is exactly the same as the one in $[15,16]$ where line signal spectra are reconstructed from compressed measurements using an off-grid approach. It was shown that $\|\mathbf{x}\|_{\mathcal{A}}$ where $\mathcal{A}$ is given by (7) can be expressed as the solution of a semidefinite program

$$
\|\mathbf{x}\|_{\mathcal{A}}=\min _{t, \mathbf{u}}\left\{\frac{1}{2} u_{1}+\frac{1}{2} t \mid\left(\begin{array}{cc}
\operatorname{toep}(\mathbf{u}) & \mathbf{x} \\
\mathbf{x}^{*} & t
\end{array}\right) \succeq 0\right\},
$$

where toep $(\mathbf{u})$ is the hermitian Toeplitz matrix whose first column is $\mathbf{u}, u_{1}$ is the first element of $\mathbf{u}, \mathbf{x}^{*}$ is the conjugate transpose of $\mathbf{x}$, and $\mathbf{B} \succeq 0$ means that the matrix $\mathbf{B}$ is positive 
semidefinite (PSD). The proof is based on the Vandermonde decomposition lemma for PSD Toeplitz matrices; see Proposition 2.1 in [16].

Now, it follows that (5) can be formulated as

$$
\min _{t, \mathbf{u}, \mathbf{x}}\left\{\frac{1}{2} u_{1}+\frac{1}{2} t \mid\left(\begin{array}{cc}
\text { toep }(\mathbf{u}) & \mathbf{x} \\
\mathbf{x}^{*} & t
\end{array}\right) \succeq 0, \mathbf{y}=\mathbf{I}_{\mathcal{S}} \mathbf{x}\right\} \text {. }
$$

Once (9) is solved, the elements of $\mathbf{x}$ yield the reconstructed values of $H_{\mathrm{RTF}}\left(\theta_{k}\right)$ for $k=0, \ldots, L-1$. As noted in Section 2, $\mathrm{x}$ is not conjugate symmetric in general. A simple way to obtain a practical RTF estimate (corresponding to a finite filter) is to symmetrize the vector according to the first half of its elements. Note, however, that this approach may bring certain loss in accuracy. An alternative way is to use properties of the dual solution of (9) that enables to identify parameters of the model (3); see Section 2.2 in [16].

\subsection{Discretized-Time Domain Reconstruction}

The other possibility to reconstruct the RTF is to find the sparsest ReIR representing the incomplete measurements in the discretized-time domain. In view of the general formulation (5), we define the atomic set as

$$
\mathcal{A}=\left\{ \pm \mathbf{e}_{k} \mid k=1, \ldots, q L\right\}
$$

where $\mathbf{e}_{k}$ denotes the $k$ th column of the $q L \times q L$ identity matrix; $q$ determines the discretization of the time axis and should be generally higher than $1 / L$ (integer values of $q$ are of practical interest). Now, the atomic norm corresponds to the $\ell_{1}$ norm (defined on $\mathcal{R}^{q L}$ ).

Compared to the previous subsection, here $\mathrm{x}$ represents the reconstructed ReIR on the time-axis discretized by factor $q L$. This inherently assumes that $h_{\text {rel }}$ has finite response of maximum length $q L$ in that domain. The value of the vector $\mathbf{y}$ is the same as in the previous section. The measuring matrix $\Phi$ is thus equal to $\mathcal{F}_{\mathcal{S}}$ where $\mathcal{F}$ is the matrix of the DFT of the length $q L$. Hence, (9) takes the form

$$
\min _{\mathbf{x} \in \mathcal{R}^{q L}}\|\mathbf{x}\|_{1} \quad \text { w.r.t. } \quad \mathbf{y}=\mathcal{F}_{\mathcal{S}} \mathbf{x}
$$

which is indeed the well-known basis pursuit program [20]. In fact, the approach proposed in [9] corresponds with the one proposed here when $q=1$.

A typical way to proceed with the solution of (11) is to resample it at $1 / q$ times back to the original discrete-time domain, thereby obtain the reconstructed $h_{\text {rel }}$ of the length $L$. By applying the DFT to the reconstructed $h_{\text {rel }}$ we arrive at the reconstructed RTF.

\section{EXPERIMENTS}

In experiments, we consider situations when the RTF is measured in ideal noise-free conditions $\left(y_{\mathrm{L}}=y_{\mathrm{R}}=0\right)$ but the training signal emitted by the target source has spectrum that does not cover the whole frequency range ${ }^{1}$. In that case, it is difficult to estimate the whole RTF. By taking only the frequencies that are sufficiently active, an incomplete RTF is obtained. The methods described in this paper are applied to reconstruct the whole RTF.

\subsection{Artificial ReiR Obeying the Model (3)}

Here, the training signal is a white Gaussian noise passed through a FIR ${ }^{2}$ band-stop filter of length 200 with cutoff band from $100 \mathrm{~Hz}$ to $f_{b} \mathrm{~Hz}$, where $f_{b}$ ranges from $200 \mathrm{~Hz}$ to $7 \mathrm{kHz}$; the duration of the signal is $1 \mathrm{~s}$; the sampling frequency is $16 \mathrm{kHz}$. The signal on the left microphone $s(n)$ is the training signal convolved with a room impulse response taken from the database [17] ( $T_{60}=160 \mathrm{~ms}$, the source-microphone distance is $1 \mathrm{~m}$, the angle is $0^{\circ}$ ). The signal on the right microphone is equal to $s(n)$ convolved with an artificial ReIR generated according to the model (3) with $L=128, S=6$ and $a_{1}=1$. The amplitudes $a_{\ell}, \ell=2, \ldots, 6$ were generated uniformly at random from $[-0.5,0.5]$ and sorted according to their magnitudes. Similarly, the delays $d_{\ell}, \ell=1, \ldots, 6$, were taken from $[0, L-1]$ but such that $\min _{k \neq \ell}\left|d_{k}-d_{\ell}\right| / L \geq$ $1 /\lfloor(L-1) / 4\rfloor$ in order to meet the condition of Theorem 1.1 in [16]. The generated ReIR is shown in Fig. 1(a).

Then, the RTF is estimated by selected methods. The results are evaluated in terms of attenuation rate (ATR) that is defined as the ratio between the average power of the signals on microphones and the power of the output of (2) where $\hat{h}$ is the inverse DFT of the estimated RTF. The testing signals are generated as described above but without applying the bandstop filter (the spectrum of the testing signal is almost flat, influenced only by the room impulse response).

The first compared method estimates $h_{\text {rel }}$ using ordinary least-squares approach (LS) minimizing $\sum_{n}\left[\left\{h * x_{\mathrm{L}}\right\}(n)-\right.$ $\left.x_{\mathrm{R}}(n)\right]^{2}$ over $h$ of length $L$. The DFT of the LS solution provides an estimate of the whole RTF. This is taken to build up an incomplete measurement as follows: The power spectrum of $x_{\mathrm{L}}(n)=s(n)$ is estimated via Welch's method (function pwelch in Matlab). Any $k \in\{0, \ldots, L / 2\}$ is put into $\mathcal{S}$ if the $k$ th frequency in $x_{\mathrm{L}}(n)$ appears to be sufficiently active (its magnitude is higher than one tenth of the maximum power over all frequencies). This selection reliably rejects frequencies that are missing in the training signal.

Then, the methods described in Section 3 are applied to reconstruct the whole RTF estimate from the incomplete measurement. The acronym "BP" is used for the methods based on the solution of (11), which we compute using the SPGL1 package $^{3}[18]$. For the solution given by (9), computed by means of SDPT3 ${ }^{4}$ [19], we use the acronym "SDP".

\footnotetext{
${ }^{1}$ This often happens, for example, when the training signal is speech.

${ }^{2}$ The filter is designed using the window method with Hamming window.

${ }^{3}$ http: // www.cs.ubc.ca/ $\sim \operatorname{mpf} / \operatorname{spg} 11$

${ }^{4}$ http://www. math. nus.edu.sg/ $\sim$ mattohkc/sdpt 3. html
} 

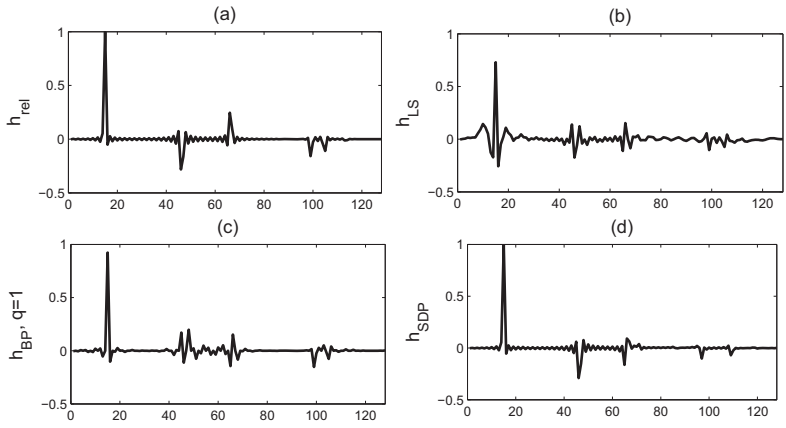

Fig. 1. (a) The original and the estimated ReIRs for $f_{b}=$ $3 \mathrm{kHz}$ by (b) LS, (c) BP with $q=1$ and (d) SDP.

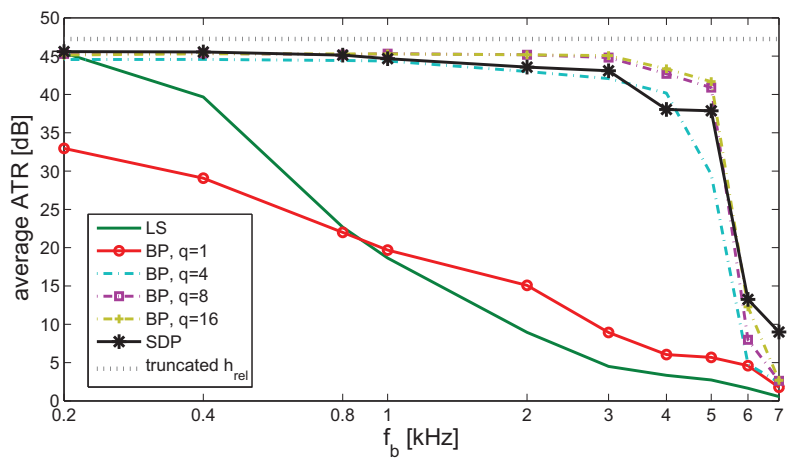

Fig. 2. Results of the first experiment in terms of ATR achieved when the target signal is white Gaussian noise. To compare, the "optimum" ATR achieved by the true $h_{\text {rel }}$ truncated to the length $L$ is included.

The results averaged over 100 trials are shown in Fig. 2. The ATRs are decreasing with growing $f_{b}$ (up to the optimum ATR by the true $h_{\text {rel }}$ truncated to length $L$ ), because the missing frequency band $\left[100, f_{b}\right] \mathrm{Hz}$ is becoming wider. The ATRs by BP with $q>1$ and by SDP are nearly or above $40 \mathrm{~dB}$ for $f_{b} \leq 4 \mathrm{kHz}$, which is very close to exact identification of $h_{r e l}$. The methods profit from the fact that $h_{\text {rel }}$ was generated according to the assumed (or approximate in case of BP) model (3). SDP is slightly outperformed by BP with $q>1$ for $f_{b} \leq 5 \mathrm{kHz}$; see the explanation of this accuracy loss at the end of Section 3.1. The experiments in [16] show that SDP can identify the parameters of the model (3) with higher accuracy than BP. Finally, it is worth to note that LS is outperformed by all the methods considered in this paper for $f_{b}>800 \mathrm{~Hz}$.

\subsection{Real-World Situation}

We conducted a similar experiment to the previous one where the training signal was speech (a female utterance taken from SiSEC $2013^{5}$ ). The signal was convolved with impulse re-

\footnotetext{
$5_{\text {http: }} / / /$ sisec.wiki.irisa.fr/tiki-index.php?page= Two-channel+mixturestof+speech+and+real-world+
}

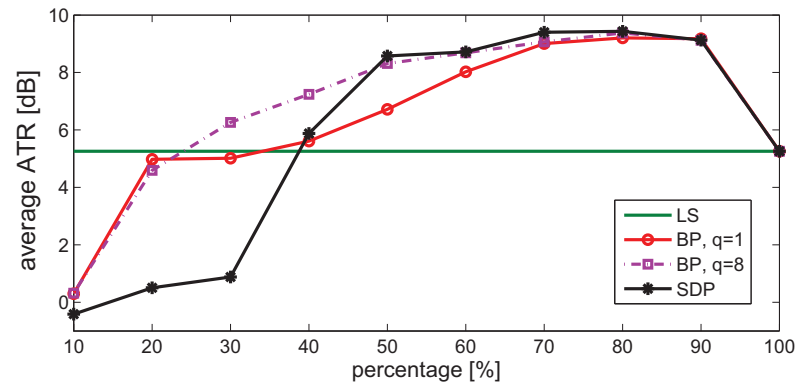

Fig. 3. Results of the second experiment in terms of average ATR. For comparison, the ATR of $13.9 \mathrm{~dB}$ was achieved by LS when computed using a white noise (the whole frequency range) training signal.

sponses from the database [17] corresponding to the target source position at $60^{\circ}$ (at the distance of $1 \mathrm{~m}$ ), by which the signals on microphones were obtained.

The signals were divided into intervals of length $1 \mathrm{~s}$. On each interval, the RTF estimate using LS ( $L=128$ ) was computed. The incomplete RTF measurement was taken by selecting $p$ percents of the most active frequencies in the leftmicrophone signal; $p$ is referred to as percentage. BP and SDP were then applied. The estimated RTFs were evaluated using a testing signal, which is $1 \mathrm{~s}$ of white Gaussian noise played from the same position as the training speech (the signal was passed through the same room impulse responses).

The results in Fig. 3 show that the best ATR is achieved by BP with $q=8$ and by SDP for the percentage ${ }^{6}$ between $70-80 \%$. LS is outperformed by the proposed methods for the percentage higher or equal to $40 \%$. This, for example, means that up to $60 \%$ of the frequency range could be occupied by noise.

The computational burden by SDP is significantly higher compared to $\mathrm{BP}$; the average running times were, respectively, about $15 \mathrm{~s}$ and $0.2-1 \mathrm{~s}$ on a PC with quad-core i7 $2.6 \mathrm{GHz}$ processor.

\section{CONCLUSIONS}

Sparse approximations of the RTF derived from its incomplete measurement have been shown to yield better ATR than the initial (complete) estimate. In future work, we will consider the de-noising formulation of (5)

$$
\min _{\mathbf{x}}\|\mathbf{x}\|_{\mathcal{A}} \quad \text { w.r.t. } \quad\|\Phi \mathbf{x}-\mathbf{y}\|_{2} \leq \epsilon
$$

with $\epsilon>0$, which might improve the current results, especially, when the incomplete measurements are noisy [15].

\footnotetext{
background+noise

${ }^{6}$ It should be noted that the optimum percentage strongly depends on the experiment, so the value $70-80 \%$ is not the optimal choice in general; see also results of experiments in [9].
} 
The simulations in the paper show that BP with sufficiently high oversampling factor $q$ provides a more practical substitute for SDP, unless faster methods for SDP exist.

\section{REFERENCES}

[1] S. Gannot, D. Burshtein, and E. Weinstein, "Signal enhancement using beamforming and nonstationarity with applications to speech," IEEE Trans. on Signal Processing, vol. 49, no. 8, pp. 1614-1626, Aug. 2001.

[2] S. Doclo and M. Moonen, "GSVD-based optimal filtering for single and multimicrophone speech enhancement," IEEE Trans. Signal Processing, vol. 50, no. 9, pp. 2230-2244, Sep. 2002.

[3] S. Gannot and I. Cohen, "Speech Enhancement Based on the General Transfer Function GSC and Postfiltering," IEEE Trans. on Speech and Audio Processing, vol. 12, No. 6, pp. 561-571, Nov. 2004.

[4] A. Krueger, E. Warsitz, and R. Haeb-Umbach, "Speech enhancement with a GSC-like structure employing eigenvector-based transfer function ratios estimation," IEEE Trans. on Audio, Speech, and Language Processing, vol. 19, no. 1, Jan. 2011.

[5] Y. Takahashi, T. Takatani, K. Osako, H. Saruwatari, K. Shikano, "Blind Spatial Subtraction Array for Speech Enhancement in Noisy Environment," IEEE Transactions on Audio, Speech, and Language Processing, vol. 17, no. 4, pp. 650-664, May 2009.

[6] K. Reindl, S. Markovich-Golan, H. Barfuss, S. Gannot, W. Kellermann, "Geometrically Constrained TRINICON-based relative transfer function estimation in underdetermined scenarios," IEEE Workshop on Applications of Signal Processing to Audio and Acoustics (WASPAA), pp. 1-4, 2013.

[7] R. Talmon and S. Gannot, "Relative transfer function identification on manifolds for supervised GSC beamformers," in Proc. of the 21st European Signal Processing Conference, Marrakech, Morocco, Sep. 2013.

[8] Z. Koldovský, J. Málek, P. Tichavský, and F. Nesta, "Semi-blind Noise Extraction Using Partially Known Position of the Target Source," IEEE Trans. on Speech, Audio and Language Processing, vol. 21, no. 10, pp. 2029-2041, Oct. 2013.

[9] Z. Koldovský, J. Málek, and S. Gannot, "Spatial Source Subtraction Based on Incomplete Measurements of Relative Transfer Function," IEEE/ACM Trans. on Speech, Audio and Language Processing, vol. 23, no. 8, pp. 1335-1347, Aug. 2015.
[10] J. Málek and Z. Koldovský, "Sparse Target Cancellation Filters with Application to Semi-Blind Noise Extraction," Proc. of the 41st IEEE International Conference on Audio, Speech, and Signal Processing (ICASSP 2014), Florence, Italy, pp. 2109-2113, May 2014.

[11] D. L. Donoho, "Compressed sensing," IEEE Transactions on Information Theory, vol. 52, no. 4, pp. 12891306, April 2006.

[12] J. B. Allen and D. A. Berkley, "Image method for efficiently simulating small-room acoustics," J. Acoust. Soc. Amer., vol. 65, no. 4, pp. 943-950, Apr. 1979.

[13] F. Antonacci, J. Filos, M. R. P. Thomas, E. A. P. Habets, A. Sarti, P. A. Naylor, S. Tubaro, "Inference of Room Geometry From Acoustic Impulse Responses," IEEE Transactions on Audio, Speech, and Language Processing, vol. 20, no. 10, pp.2683-2695, Dec. 2012.

[14] V. Chandrasekaran, B. Recht, P. Parrilo, and A. Willsky, "The Convex Geometry of Linear Inverse Problems," Foundations of Computational Mathematics, vol. 12, no. 6, pp. 805-849, 2012.

[15] B. N. Bhaskar, T. Gongguo, and B. Recht, "Atomic Norm Denoising With Applications to Line Spectral Estimation," IEEE Transactions on Signal Processing, vol. 61, no. 23, pp. 5987-5999, Dec. 2013.

[16] T. Gongguo, B. N. Bhaskar, P. Shah, B. Recht, "Compressed Sensing Off the Grid," IEEE Transactions on Information Theory, vol. 59, no. 11, pp. 7465-7490, Nov. 2013.

[17] E. Hadad, F. Heese, P. Vary, and S. Gannot, "Multichannel audio database in various acoustic environments," International Workshop on Acoustic Signal Enhancement 2014, Antibes, France, Sept. 2014.

[18] E. van den Berg and M. P. Friedlander, "Probing the Pareto frontier for basis pursuit solutions," SIAM J. on Scientific Computing, vol. 31, no. 2, pp.890-912, Nov. 2008.

[19] K. C. Toh, M. J. Todd, and R. H. Tütüncü, "SDPT3 - a Matlab software package for semidefinite programming," Optimization Methods and Software, vol. 11, pp. 545-581, 1999.

[20] S. S. Chen, D. L. Donoho, M. A. Saunders, "Atomic Decomposition by Basis Pursuit", SIAM Journal on Scientific Computing, Vol. 20, No. 1., pp. 33-61, 1999. 